\title{
Reflets
}

Revue d'intervention sociale et communautaire

\section{L'accès aux services sociaux et de santé en français et la formation des professionnelles et des professionnels en situation francophone minoritaire canadienne}

\author{
Marie Drolet, Claire-Jehanne Dubouloz et Josée Benoît
}

Volume 20, numéro 2, automne 2014

L'accès aux services sociaux et de santé en français et la formation des professionnelles et professionnels en situation francophone minoritaire canadienne

URI : https://id.erudit.org/iderudit/1027584ar

DOI : https://doi.org/10.7202/1027584ar

Aller au sommaire du numéro

Éditeur(s)

Reflets, Revue d'intervention sociale et communautaire

ISSN

1203-4576 (imprimé)

1712-8498 (numérique)

Découvrir la revue

Citer ce document

Drolet, M., Dubouloz, C.-J. \& Benoît, J. (2014). L'accès aux services sociaux et de santé en français et la formation des professionnelles et des professionnels en situation francophone minoritaire canadienne. Reflets, 20(2), 10-19.

https://doi.org/10.7202/1027584ar

Tous droits réservés (C Reflets, Revue d’intervention sociale et communautaire, 2014

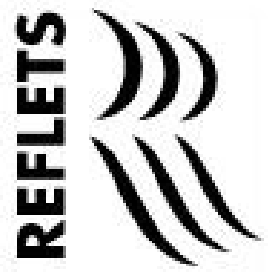




\section{L'accès aux services sociaux et de santé en français et la formation des professionnelles et des professionnels en situation francophone minoritaire canadienne}

Marie Drolet, Ph. D., Professeure titulaire École de service social, Université d'Ottawa

Claire-Jehanne Dubouloz, Ph. D., Professeure titulaire École des sciences de la réadaptation, Université d'Ottawa Josée Benoit, Ph. D., associée principale de recherche Groupe de recherche sur la formation professionnelle en santé et service social en contexte francophone minoritaire (GReFoPS), Université d'Ottawa

Au cours des cinq dernières années, quatre revues scientifiques ont consacré de leurs numéros à la recherche sur la santé des communautés francophones en situation minoritaire au Canada. D'abord, sur la lancée du $2^{\text {e }}$ Forum national de recherche sur la santé des francophones en situation minoritaire organisé en 2007 par le Consortium national de formation en santé (CNFS) et ses partenaires, la revue Francophonie d'Amérique ( $\left.\mathrm{N}^{\circ} 28\right)$ publiait en 2009 un tel numéro sous la direction d'Éric Forgues, de Josée Guignard Noël et de Rodrigue Landry, une équipe réunie par l'Institut canadien de recherche sur les minorités linguistiques. 
Les articles portaient sur les trois grands axes de recherche du CNFS, soit les déterminants de la santé; la gouvernance, la gestion et la prestation des services en français; et les liens qui unissent la langue, la culture et la santé. Comme le mentionnent Forgues, Guignard Noël et Landry (p.12), et ce constat est toujours aussi pertinent aujourd'hui, "la recherche se trouve [...] au cœur de la vitalité et du mieux-être » de nos communautés francophones linguistiquement minoritaires.

Puis en 2012, Reflets (Vol. 18, № 2) consacrait un numéro thématique aux inégalités de santé touchant les communautés francophones en situation minoritaire au Canada. Dirigé par un regroupement interinstitutionnel de l'Université Laurentienne (Monique Benoît, avec la collaboration de Marie-Luce Garceau), de l'Université d'Ottawa (Louise Bouchard) et de l'Université de la Saskatchewan (Anne Leis), ce recueil de recherches inédites se voulait " une contribution à la prise de conscience et à la nécessité de réduire les inégalités sociales de santé qui affectent la minorité linguistique "(p. 13). Les articles abordent l'offre active de services en français, les perceptions des professionnelles et des professionnels de la santé et des services sociaux quant aux difficultés d'accès à des soins et services en français et de qualité au Manitoba, les services offerts aux personnes âgées au NouveauBrunswick, ainsi que dans le champ de la santé mentale dans l'Est ontarien et chez les anglophones minoritaires québécois.

En 2013, la Revue canadienne de santé publique (Vol. 104, № 6) diffusait un supplément dirigé par Anne Leis et Louise Bouchard sur l'état de santé des communautés de langue officielle vivant en contexte minoritaire. Ce numéro faisait état des travaux du Réseau de recherche interdisciplinaire sur la santé des francophones en situation minoritaire au Canada, du Réseau de recherche appliquée sur la santé de la population francophone de l'Ontario et de l'Institut de recherche de l'Hôpital Montfort. Ce supplément cherchait à réaliser " une lecture plus approfondie des enjeux de santé en situation minoritaire" (p. S3).

Enfin, un numéro spécial de la revue Minorités linguistiques et société/Lingusitic Minorities and Society, lequel reste à paraitre sous la direction de Suzanne Dupuis-Blanchard et de Sylvain Vézina 
(Université de Moncton), regroupe des articles issus du $3^{\text {e }}$ Forum national de recherche sur la santé des francophones en situation minoritaire organisé en juin 2012 par le CNFS Secrétariat national et ses 11 partenaires institutionnels. Ce numéro vise à ce que la recherche œuvre au profit d'un meilleur accès aux services sociaux et de santé en français en contexte minoritaire. Les deux thèmes exploités, recherche et accès, soulèvent une problématique complexe et multidimensionnelle arborant des perspectives à la fois d'ordre médical, organisationnel, sociopolitique, culturel ou autre.

À partir de quelques études récentes dans le domaine, établissons certains constats solides sur lesquels inscrire nos futures réflexions. Bouchard et Desmeules (2013) mettent très clairement en valeur la place désavantageuse qu'ont les francophones vivant en situation minoritaire par rapport aux anglophones majoritaires demeurant dans les mêmes régions du pays. Ces francophones se perçoivent tout d'abord en moins bonne santé que leurs concitoyens anglophones (16,65\% contre 13,14\%). La population francophone est globalement plus âgée : 19,46\% des francophones minoritaires ont 65 ans et plus contre $17,74 \%$ chez les anglophones. Leur niveau de scolarité demeure toujours inférieur, $20,73 \%$ des francophones minoritaires n'ayant pas terminé leurs études secondaires contre 12,39 \% chez les anglophones des mêmes provinces. Quant au revenu, l'écart défavorable est moindre, 20,43\% des francophones ayant un faible revenu, soit à l'intérieur des 20 premiers percentiles, contre 17,28 \% chez les anglophones. Proportionnellement, les francophones en situation minoritaire vivent beaucoup plus en milieu rural, en régions plus éloignées et de façon plus dispersée (29, $53 \%$ contre 17,75\%). Dans le supplément de la Revue canadienne de santé publique de 2013, certains chercheurs font d'autres constats dérangeants. Nous faisons moins d'activités physiques durant nos loisirs que les anglophones majoritaires (49,1\% contre 47, $2 \%$ ) (Imbeault, et collab., 2013). Il y a une plus grande prévalence de surpoids et d'obésité chez les francophones en contexte minoritaire, ce qui va dans le sens des données socio-économiques décrites cidessus, la minorité francophone faisant plus partie des milieux 
et des groupes vulnérables, soit de ceux qui ont plus tendance à l'embonpoint (Gagnon-Arpin, et collab., 2013).

De surcroît, en matière d'accès à des services sociaux et de santé dans leur langue, $75 \%$ des francophones en contexte minoritaire affirment qu'il est important pour eux de recevoir leurs services sociaux et de santé en français; en revanche, seulement $40 \%$ d'entre eux disent utiliser le français avec divers professionnelles ou professionnels de la santé et des services sociaux (GagnonArpin, 2011). À partir d'une recherche qualitative tenue auprès d'intervenantes et d'intervenants de la santé et des services sociaux de l'Est ontarien, Drolet, et collab. (2014) mettent en lumière des trajectoires discontinues de services en français. En contrepartie, les professionnelles ou professionnels francophones agissent souvent comme des personnes pivots. Leurs réseaux de contacts permettent aux personnes et à leur famille de dépasser ces défis d'accès (Savard, et collab., sous presse). Les mêmes situations se présentent au Manitoba, en plus d'une pénurie marquée de médecins de famille, de pharmaciens et de soutien communautaire pour les personnes touchées et leurs proches aidants qui désirent un service en français sur un continuum (de Moissac, et collab., 2012). Au Nouveau-Brunswick, dans une étude sur les enjeux du maintien à domicile des aînés francophones (2013), DupuisBlanchard et Villalon constatent que ces services doivent être mieux ajustés au quotidien complexe de la minorité linguistique. Bref, les défis d'accès prennent des angles moins visibles, mais ils sont toujours présents.

Par conséquent, l'appartenance à un groupe linguistique minoritaire est maintenant de plus en plus reconnue comme un déterminant dans le domaine des services sociaux et de la santé (Bouchard, et collab., 2009; Bouchard et Desmeules, 2013; Leis et Bouchard, 2013), surtout que la communication verbale s'avère tellement à la base de l'intervention à l'intérieur de ces champs de pratique. Il est également établi que les professionnelles ou professionnels nouveaux de ces domaines, même ceux formés en français, ne sont souvent pas adéquatement outillés pour œuvrer auprès des communautés francophones en situation linguistique minoritaire tant pour reconnaître les enjeux liés à l'offre active 
de services en français que pour poser des gestes concrets afin d'améliorer l'accès auxdits services (Bouchard et Vézina, 2009).

En complément, et de façon très éloquente, dans le numéro de Reflets mentionné ci-dessus (2012, p. 35), M. Hubert Gauthier, le $1^{\text {er }}$ président de la Société santé en français et son directeur général de 2005-2009, concluait son entrevue sur cette question : «Y a-t-il autre chose qu'on peut faire pour améliorer l'état de santé de la population francophone et réduire les écarts entre la communauté minoritaire et la majoritaire? "C'est en se basant sur cet appel percutant à continuer notre réflexion, à approfondir les enjeux en la matière et à se mobiliser pour l'action que le présent numéro de Reflets s'arrime aux ouvrages déjà publiés en s'inscrivant dans leur lignée. Son objectif est de partager des recherches et des pratiques novatrices, de favoriser l'interdisciplinarité et de mettre en commun des expertises complémentaires pour cerner les avancées en matière d'accès à des services sociaux et de santé en français et de formation des professionnelles ou des professionnels dans ces domaines en francophonie minoritaire canadienne, et ce, en vue d'encore mieux intervenir.

Dans le premier des quatre articles du Dossier, Simon Lapierre, Cécile Coderre, Isabelle Côté, Marie-Luce Garceau et Chantale Bourassa diffusent les résultats d'une recherche qualitative réalisée en Ontario et au Nouveau-Brunswick sur l'accès aux services en français pour les femmes francophones victimes de violence conjugale et leurs enfants. Ces données mettent en relief les difficultés souvent rencontrées en matière d'accès à des services sociaux et de santé en français en milieu francophone minoritaire. L'équipe souligne entre autres des lacunes dues au manque de disponibilités de services en français pour des services spécialisés ou des groupes d'appui, ainsi que des transferts à des ressources en anglais. Les femmes victimes de violence conjugale et leurs enfants étant confrontés à des réalités particulières, ces lacunes dans l'accès aux services en français compromettent clairement leur bien-être, leur santé et leur sécurité, les obligeant, faute de services, à demeurer parfois avec un conjoint violent. Devant ces impacts majeurs, le manque d'accès aux services en français devient une forme de revictimisation des femmes. 
Louis Giguère et Brian Conway explorent de leur côté le potentiel de services médicaux offerts dans les langues minoritaires en Colombie-Britannique. Leur étude révèle que des médecins non francophones parlant français génèrent une retombée linguistique qui amplifie de façon remarquable l'offre potentielle de services de santé et de services sociaux en français. Ce mécanisme structurant pourrait s'appliquer aux autres professions de la santé et des services sociaux partout au Canada. De surcroît, cet impact potentiel positif serait plus prononcé quand le contexte est davantage minoritaire. Les auteurs appellent les communautés francophones à mieux identifier, engager et outiller les professionnelles ou professionnels "francophiles"; ces communautés doivent élaborer une logique novatrice pour dégager le potentiel d'offre de services de santé et de services sociaux en français chez les "francophiles " dans un contexte multilingue ou tout milieu affrontant une pénurie de personnel francophone.

L'article signé par Jacinthe Savard, Lynn Casimiro et Josée Benoît (École des sciences de la réadaptation de l'Université d'Ottawa) et Pier Bouchard (Université de Moncton) cherche à répondre à la question suivante : Comment peut-on mesurer l'impact des actions entreprises pour améliorer l'offre active de services sociaux et de santé en français dans les communautés francophones en situation minoritaire? Y sont décrits les premiers pas d'une démarche visant à mesurer l'évolution des comportements d'offre active à la suite d'activités de formation ou de changements organisationnels en faveur de services en français. Une telle mesure est aussi susceptible d'être utile dans les recherches visant à saisir les déterminants de ces comportements d'offre active.

L'article de Claire-Jehanne Dubouloz, Josée Benoît, Paulette Guitard, Lucie Brosseau, Jacinthe Savard et Lucy-Ann Kubina (École des sciences de la réadaptation) et de Marie Drolet (École de service social de l'Université d'Ottawa) présente des lignes directrices de la formation des futurs professionnelles et professionnels des services sociaux et de santé dans le but d'inclure l'apprentissage du concept d'offre active de services en français 
dans les programmes universitaires et collégiaux de formation. Fondées sur une recension d'écrits et les résultats d'un sondage réalisé auprès du corps professoral des programmes francophones de santé et de service social au Canada, ces lignes directrices identifient les appuis nécessaires au niveau de l'institution, des facultés, des programmes d'études et du corps professoral pour une intégration du concept d'offre active de services sociaux et de santé en français.

Dans la rubrique Des pratiques à notre image, six articles reprennent à leur manière les enjeux reliés à l'accès aux services sociaux et de santé en français en contexte francophone minoritaire tout en proposant et en décrivant des pratiques exemplaires d'offre de services en français.

Un article porte sur le Consortium national de formation en santé (CNFS) et sur son mandat envers la formation à l'offre active de services de santé et services sociaux en français. Il propose une définition large de l'offre active de même que des pratiques exemplaires visant à conscientiser les futurs professionnelles ou professionnels de la santé et des services sociaux aux enjeux et aux défis liés à la prestation des services en français et à mieux les outiller pour intervenir auprès des populations francophones vivant en contexte minoritaire. Un aperçu de la vision d'avenir du CNFS couronne le tout.

Un autre article porte sur la Société Santé en français (SSF), un organisme pancanadien à but non lucratif qui veille à assurer un meilleur accès à des services de santé et des services sociaux de qualité en français. On y définit d'abord son mandat et sa composition; on y soulève ensuite l'importance du réseautage en santé, les progrès qui ont été réalisés depuis la mise sur pied de cet organisme, l'importance de la langue dans l'accès aux services sociaux et de santé et le rôle des ressources humaines. En 2012, la Société Santé en français s'est dotée d'un nouveau plan directeur quinquennal intitulé Destination Santé 2018. Les quatre champs prioritaires de ce plan sont déployés dans l'article.

Yalla Sangaré (Université Sainte-Anne en Nouvelle-Écosse) offre dans son article un exemple de pratique exemplaire 
et innovatrice, soit le Centre de santé de Clare, un Centre multiservice pour les régions rurales francophones éloignées. L'expérience de ce centre dans son offre de services de santé en français peut servir de modèle pour d'autres communautés francophones vivant en contexte semblable.

Marie-Pier Audet et Marie Drolet discutent des services en santé mentale pour les adolescentes et adolescents francophones de la région d'Ottawa. En dépit de la complexité et de la difficulté d'accès à des services de santé mentale pour ces jeunes, les résultats de leur recherche démontrent que certains services déjà en place sont remarquables. Les auteures soulignent le rôle important que jouent le Centre psychosocial et le travail social en milieu scolaire pour les jeunes francophones de la région d'Ottawa vivant en situation minoritaire et présentant des problèmes de santé mentale.

Roger Parent, Louise Bouchard et Marc Lebel présentent ensuite un projet de formation interculturelle adaptée aux collèges francophones canadiens. Le projet Aînés francophones et intervenants multiethniques en santé veut documenter et analyser en fonction des compétences culturelles acquises la portée à moyen terme d'une session de formation interculturelle qui vise les étudiantes et les étudiants des collèges qui veulent devenir préposés aux bénéficiaires dans les centres de soins de longue durée. Les personnes rencontrées étaient en général d'immigration récente. Par l'entremise de la cartographie conceptuelle, les chercheurs soulèvent une élaboration progressive de la compétence culturelle, telle que vécue et exprimée par les participantes et participants au projet.

Enfin, l'article de Corine Carbonneau et Marie Drolet expose les expériences de personnes âgées francophones vivant en situation linguistique minoritaire et atteintes d'une démence et certaines des particularités de leur trajectoire au sein des services sociaux et de santé, et ce, selon la perspective de proches aidantes ou aidants. À partir des résultats de leur étude, les auteures soulèvent l'importance du partenariat entre les multiples paliers d'intervention voués au bien-être de personnes âgées devenues vulnérables et proposent une meilleure intégration des services sociaux et de santé en français. 
En somme, nous vous invitons à apprécier ces quelques avancées touchant l'accès à des services en français en contexte minoritaire et la formation en la matière. Nous visons également à ce que nous réfléchissions ensemble aux défis qui persistent et à éveiller votre engagement. Ces défis encore nombreux nécessitent très nettement que nous nous unissions pour agir, pour former en ayant un solide ancrage avec les milieux de pratique et pour revendiquer des politiques en faveur d'un meilleur accès à des services sociaux et de santé en français, de qualité et sécuritaires.

\section{Bibliographie}

BOUCHARD, Louise, et Martin DESMEULES (2013). "Les minorités linguistiques du Canada et la santé ", Healthcare Policy / Politiques de Santé,Vol. 9, p. S38-S47.

BOUCHARD, Louise, Isabelle GABOURY, Marie-Hélène CHOMIENNE, Anne GILBERT et Lise DUBOIS (2009). "La santé en situation linguistique minoritaire ", Longwoods Publishing, Vol. 4, No 4, p. 36-42. doi:10.12927/hcpol.2009.20807

BOUCHARD, Pier, et SylvainVÉZINA (2009). L'outillage des étudiants et des nouveaux professionnels : un levier essentiel à l'amélioration des services de santé en français, [rapport de recherche], Ottawa, Ontario, Consortium national de formation en santé (CNFS), $66 \mathrm{p}$.

DE MOISSAC, Danielle, Janelle DE ROCQUIGNY, Florette GIASSON, Cindy-Lynne TREMBLAY, Natalie AUBIN, Marc CHARRON et Gratien ALLAIRE (2012). "Défis associés à l'offre de services de santé et de services sociaux en français au Manitoba : perceptions des professionnels », Consortium Erudit,Vol. 18, № 2, p. 66-100. doi:10.7202/1013174ar

DROLET, Marie, Jacinthe SAVARD, Josée BENOÎT, Isabelle ARCAND, Sébastien SAVARD, Josée LAGACÉ, Sylvie LAUZON et Claire-Jehanne DUBOULOZ (2014). «Health Services for Linguistic Minorities in a Bilingual Setting: Challenges for Bilingual Professionals ", SAGE Publications, Vol. 24, №3, p. 295-305. doi:10.1177/1049732314523503

DUPUIS-BLANCHARD, Suzanne M., et Lita VILLALON (2013). " La perception des aînés francophones en situation minoritaire face aux défis et aux enjeux liés au maintien à domicile en milieu urbain néo-brunswickois ", Canadian Journal of Public Health / Revue canadienne de santé publique, Vol. 104, No 6, p. S71-S74.

GAGNON-ARPIN, Isabelle (2011). Access to Health Care Services and Self-Perceived Health of Canada's Official-Language Minorities, University of Ottawa.

GAGNON-ARPIN, Isabelle, Ewa MAKVANDI, Pascal IMBEAULT, Malek BATAL et Louise BOUCHARD (2013). "Le surplus de poids chez les francophones et les anglophones ", Canadian Journal of Public Health/ Revue canadienne de santé publique,Vol. 104, No 6 Suppl. 1, p. S21-S25.

IMBEAULT, Pascal, Ewa MAKVANDI, Malek BATAL, Isabelle GAGNON-ARPIN, GRENIER, Jean, CHOMIENNE, Marie-Hélène, BOUCHARD, Louise (2013). «Physical inactivity among 
francophones and anglophones in Canada ", Canadian Journal of Public Health/ Revue canadienne de santé publique, Vol. 104, Nº, p. S26-S30.

LEIS, Anne, et Louise BOUCHARD (2013). «Éditorial », Canadian Journal of Public Health/ Revue canadienne de santé publique, Vol. 104, № 6, supplément 1.

SAVARD, Sébastien, Isabelle ARCAND, Marie DROLET, Josée BENOÎT, Jacinthe SAVARD et Josée LAGACÉ «Les professionnels de la santé et de services sociaux intervenant auprès des francophones minoritaires : l'enjeu du capital social ", Francophonies d'Amérique, Vol. 36, (Sous presse). 